\title{
Dynamic changes of viral load and the duration of viral shedding in patients with hand, foot and mouth disease: a protocol for longitudinal study
}

\author{
Xiaoxia Duan ${ }^{1 \dagger}$, Chaoyong Zhang ${ }^{2 \dagger}$, Zhenhua Chen ${ }^{3 \dagger}$, Juan Liao ${ }^{4,5 \dagger}$, Yilan Zeng ${ }^{2}$, Weiwei Huang ${ }^{3}$, Xueling Ren ${ }^{1}$, \\ Xueqin Tang ${ }^{1}$, Hongxia Peng ${ }^{1}$, Delan Zhang ${ }^{2}$, Xiao Wang ${ }^{1}$, Ping Yuan ${ }^{1}$ and Lu Long ${ }^{1 *}$
}

\begin{abstract}
Background: The duration of virus shedding is necessary for determining the infectious period. But there were few quantitative studies on the changes of viral load and the law of the viral shedding in hand foot and mouth disease (HFMD) patients has not yet been clarified.

Methods: This study will prospectively recruit coxsackievirus A10 (CV-A10), coxsackievirus A16 (CV-A16) and coxsackievirus A6 (CV-A6) infected inpatients from January 2022 to December 2022. A series of samples and questionnaire information will be collected regularly to establish the dynamic function relationship between time and viral load changes and a Bayesian multilevel model will be constructed to clarify the evolvement rules which reflect the dynamic changes of viral load and the duration of viral shedding in patients with HFMD.

Discussion: The results of this study is expected to further clarify the evolvement rules which reflect the dynamic changes of viral load and the duration of viral shedding in HFMD patients under the influence of related factors. It can also provide important evidence for the scientific definition of the infectious period and isolation period of HFMD in China.
\end{abstract}

Keywords: Hand foot and mouth disease, Enterovirus, Viral shedding, Viral load, Bayesian multilevel model

\section{Background}

Hand, foot and mouth disease (HFMD) is an acute infectious disease caused by a group of enteroviruses. Enterovirus A71 (EV-A71) and coxsackievirus A16 (CV-A16) were the most common viral types in the past few decades [1]. After inactivated EV-A71 vaccines gradually

\footnotetext{
*Correspondence: longlu201609@163.com

†Xiaoxia Duan, Chaoyong Zhang, Zhenhua Chen, and Juan Liao have contributed equally

${ }^{1}$ Department of Epidemiology and Health Statistics, West China School of Public Health and West China Fourth Hospital, Sichuan University, No.16, Section 3, Renmin south road, Wuhou District, Chengdu, Sichuan, China

Full list of author information is available at the end of the article
}

expanded, CV-A16, coxsackievirus A6 (CV-A6) and coxsackievirus A10 (CV-A10) gradually becoming the predominant and co-circulated serotypes [2-4], and the major causative agents of outbreaks and epidemics around the world of HFMD [5-7]. Recently, the incidence of HFMD has declined, but it still ranked second in the number of in Category $\mathrm{C}$ infectious diseases in China [8].

No established treatment is available for HFMD [9], as the highly contagious HFMD spreads rapidly among children and always spreads in kindergartens, schools and other collective units, and isolation is the most effective way to control its spread. Therefore, most countries in 
the Asia-Pacific region, have adopted social distancing measures, such as closures of daycare centers and schools [10]. However, the isolation regulations for the source of infection vary greatly in different countries and regions, and there is no clear definition of the period of infection $[10,11]$. The infectious period mainly determined by the duration of virus shedding is an important basis for determining the isolation period of patients with HFMD.

Several small-scale studies have qualitatively explored the duration of viral shedding for HFMD patients induced by EV-A71 and CV-A16, but the results were quite different $[12,13]$. In addition, there were few studies that focused on CV-A6 and CV-A10. A previous meta-analysis has showed that most cured HFMD cases continued to excrete the virus for a long time, and there were large differences between individuals [14]. The current isolation period may be extremely limited in blocking the transmission of HFMD $[12,15]$, and the arbitrary extension of the HFMD isolation period is not only unscientific but will cause significant social impact [16].

This study will prospectively recruit CV-A10, CV-A16 and $\mathrm{CV}-\mathrm{A} 6$ infected inpatients as the research subjects. A series of samples and questionnaire information will be collected regularly to establish the dynamic function relationship between time and viral load changes. Bayesian multilevel model will be used to clarify the evolvement rules which reflect the dynamic changes of viral load and the duration of viral shedding in HFMD patients under the influence of related factors.

\section{Aims and hypotheses}

We aim to perform a prospective study base on patients hospitalized in the public clinical center of Chengdu to collect a series of samples from patients with HFMD induced by $\mathrm{CV}-\mathrm{A} 10, \mathrm{CV}-\mathrm{A} 16$ and $\mathrm{CV}-\mathrm{A} 6$ and evaluate the dynamic changes of viral load and the duration of viral shedding in HFMD patients under the influence of related factors. The results of the study can further provide important evidence for the scientific definition of the infectious period and isolation period of HFMD in China.

\section{Methods}

\section{Study design}

This is a hospital-based prospective cohort study, and study methodological design will follow the requirements of 'Strengthening the Reporting of Observational Studies in Epidemiology' (STROBE) checklist for cohort studies [17]. During February 1 to December 31, 2022, we will seek to enroll and trace hospitalized HFMD patients in a designated referral hospital. The demographic and clinical data of HFMD patients during the whole clinical course and series clinical specimens will be collected to evaluate the dynamic changes of viral load and the duration of viral shedding.

\section{Study site}

The study will be conducted at the public health clinical center of Chengdu, capital city of Sichuan Province in Western China. It is a designated hospital for the diagnosis and treatment of HFMD in Sichuan Province, as well as a referral hospital for critically ill cases. In addition to general enterovirus testing, all hospitalized patients tested for EV-A71, CV-A16, CV-A6 and CV-A10 serotypes to confirm diagnosis since 2018. There were on average 1500 hospitalized HFMD cases annually before 2019, which provides sample size guarantee for the smooth development of the project.

\section{Study subjects}

All hospitalized patients meeting the following inclusion and exclusion criteria during February 1 to December 31, 2022 will be recruited in this study. The initial minimum sample size of this study is expected to reach about $120-160$ cases. If the sample does not meet the minimum required follow-up volume, we will appropriately extend the project time.

\section{Inclusion criteria}

(1) Admitted to public clinical center of Chengdu due to HFMD induced by CV-A6, CV-A10 and CV-A16.

(2) Written informed consent from a parent or legal guardian for/and children.

\section{Exclusion criteria}

(1) Discharge from hospital within $24 \mathrm{~h}$ of admission.

(2) Patients from the suburbs of each district/county in the third circle of Chengdu and refuse to go to the hospital for follow-up.

\section{Sample size calculation}

The sample size for this study is $120-160$ within a period of 1 year, based on the inclusion rate of HFMD hospitalized patients in previous longitudinal studies [18], the number of hospitalized patients with HFMD in 2020, and a 20 percent loss to follow-up. In addition, according to previous studies, the multi-level model could guarantee relatively accurate parameter estimations when the high level sample is not less than $50[19,20]$. 


\section{Study procedure}

Study subjects enrolled for this study need to conduct the procedures as below. Figure 1 provides an overview of the study procedure.

\section{Enrollment of subjects}

The throat swabs of all HFMD cases will be collected on the day they are admitted to clinical public center of Chengdu to conduct qualitative PCR detection to determine virus types. Every patients singly induced by CV-A6, CV-A10 or CV-A16 will be approached after laboratory determined as soon as possible. All patients providing with written informed consent from their parent or legal guardian and/or themselves will be included in the study. We will uniformly code all patients who voluntarily participate in this project.

\section{Clinical data collection}

A structured hospitalization questionnaire including four parts will be used to collect detailed clinical data, which spans the length of a typical course of illness. In the first part, baseline data including demographics, medical

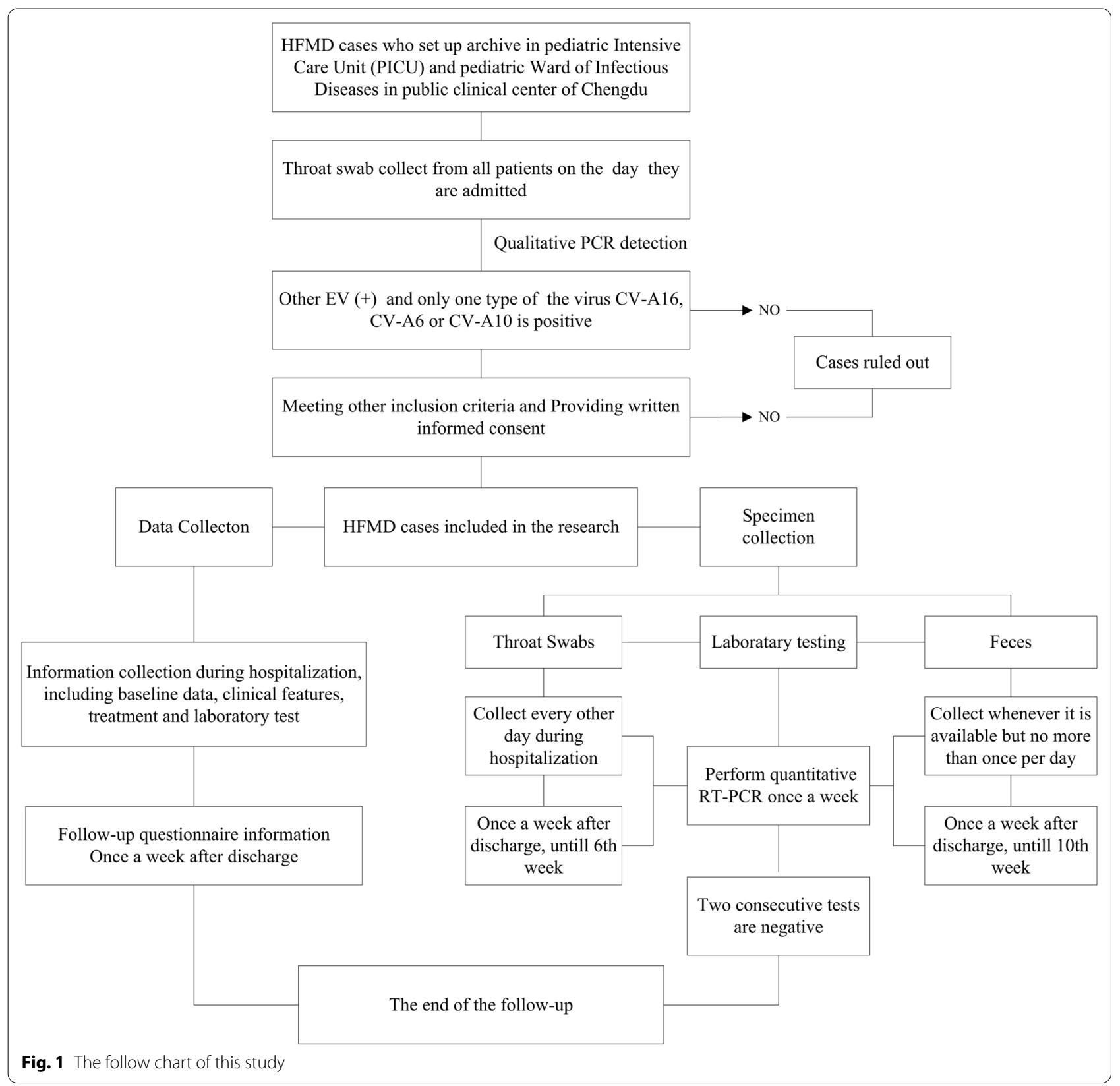


history, birth history, symptoms and signs at the onset, treatment before admission and history of EV-A71 vaccination will be collected through interview of the patients and their parents. In the second part, clinical data including detailed clinical features and complications will be collected through ward rounds, and updated every day during hospitalization. The last two parts are clinical laboratory tests and treatments, which will be exported and collected from the electronic medical record system after patients discharge from the hospital. The data collection will begin as soon as possible after the cases enter the study.

After discharge, a structured follow-up questionnaire is used to investigate the patient's health status since the last follow-up, including the occurrence of suspected HFMD symptoms, medical history, diagnosis results, and the occurrence of other HFMD cases in the same family.

\section{Clinical specimen collection}

During hospitalization, throat swabs will be collected once every other day, while feces will be collected whenever it is available but no more than once per day as long as the volume meets the requirement. After discharge, we will conduct a home follow-up to collect throat swabs and feces once a week until two consecutive test results are negative, or reach the end of follow-up time (6th week after admission for throat swabs and $10^{\text {th }}$ week for feces). All specimens will be collected according to the operating standards of the National Center for Disease Control and Prevention. All throat swabs and stool will be coded and stored in the $4{ }^{\circ} \mathrm{C}$ refrigerator temporarily (no more than $24 \mathrm{~h}$ ) before transferred to $-80^{\circ} \mathrm{C}$ freezer.

\section{Laboratory test \\ Viral RNA extraction}

The preprocessing of all the clinical specimens and extraction of viral nucleic acid will perform in Level 2 Biosafety laboratory (BSL-2). For throat swabs samples, Mix the virus sampling tube on a vortex shaker for at least $10 \mathrm{~s}$ to wash off the adhering virus or virus-containing cells, the mixture will be taken directly for RNA extraction. For stool samples, a stool suspension will be made by adding a bean size feces in to $700 \mu \mathrm{L}$ normal PBS buffer and mechanically oscillate for $30 \mathrm{~s}$ to make the sample fully mixed. The suspension will be then centrifuged at 12,000 $\mathrm{rmp}$ for $5 \mathrm{~min}$, and the upper suspension will be used for RNA extraction. ABT Viral RNA extraction Kit (ABT, Beijing, China) will be used for RNA extraction in the development of the assay and RNA extraction from clinical specimens according to the manufacturer's instructions. The extracted viral RNAs will be immediately used for real-time fluorescence quantitative PCR detection. If not, the RNAs will be eluted with $40 \mu \mathrm{l}$ of diethyl pyro carbonate-treated water and kept at $-80{ }^{\circ} \mathrm{C}$ until further use for real-time RT-PCR.

\section{Real-time fluorescence quantitative $P C R$}

The viral load of CV-A6, CV-A10 and CV-A16 will be detected by real-time fluorescence quantitative reverse transcription polymerase chain reaction (RT-PCR). The RT-PCR kit is produced by ABT Biotechnology Company (Beijing, China), the experimental operation will be carried out according to the manufacturer's instructions. The instrument is BIO-RAD CFX96 real-time fluorescence quantitative PCR instrument.

\section{Outcome measures \\ Primary outcome}

The primary outcome of the study is the dynamic changes of viral load and the duration of viral shedding in HFMD patients under the influence of related factors. To obtain this result, we will first identify the positive rate and virus load of the specific serotype enterovirus at each collection time points and the time of turning negative of HFMD patients. Then, we will use Bayesian multilevel model to fit the influence of nested fixed and random factors on the variation of viral load.

\section{Secondary outcomes}

The secondary endpoints include number of days spent in hospital, the proportion of clinical manifestation and their duration, as well as the epidemic features of hospitalized HFMD patients induced by CV-A6, CV-A10 or CV-A16.

\section{Statistical analysis}

We will use epidata3.1 to enter the data and R4.0.1 to conduct statistical analysis. We will follow the "Reporting of studies Conducted using 'Observational Routinely Collected Health Data' Statement” [21]. Our primary analysis will be the dynamic changes of viral load and the duration of viral shedding in HFMD patients, while clinical features and epidemic features of hospitalized HFMD cases will be secondary analysis.

\section{Baseline characteristics}

For continuous variables, we will summarize them using means \pm standard deviation and the comparison between the two groups will use independent sample t-test if the data conform the normal distribution. If not, we will describe interquartile ranges, and nonparametric test will be used to compare the two groups. For categorical variables, we will report data with proportion (\%) and compare groups through standard Pearson's, or Fisher's exact test if either group contains less than 10 patients. 
or Fisher's exact test if either group contains less than 10 patients. The level of significance will be set at $\mathrm{p}<0.05$.

\section{Bayesian multilevel model}

Bayesian multilevel model will be used to reflect the dynamic changes of viral load and the duration of viral shedding in HFMD patients under the influence of related factors. Because of the structural nesting and unbalanced characteristics of the research data, we will use Bayesian multilevel model to fit the influence of nested fixed and random factors on the variation of viral load.

\section{Data management and publication}

Initial study data collection is expected to be completed in late 2022. De-identified data is expected to be available a month after that. All data will be collected through paper questionnaires and entered manually by the research team. The database will be stored in an encrypted manner with Epidata3.1 limited to authorized personnel only, and the paper questionnaire will be sealed in the project office. Scientific publications or reports will not identify individual participants.

Results of the study will be submitted for publication in international peer reviewed biomedical journals. Position of authorship will be determined according to international standards for authorship in peer-reviewed journals.

\section{Study status}

All implementation site and laboratory preparations have been completed. Pre-investigation is currently conducting and will be finished on January 31, 2022, and the samples collected in pre-investigation will be used to laboratory operation process training. Formal investigation and participant recruitment will be carried out from February 1 to December 31, 2022.

\section{Discussion}

This protocol describes a longitudinal study which aims to assess the law of the viral shedding in HFMD patients. Until now, there were few quantitative studies on the changes of viral load and the law of the viral shedding in difference specimens of HFMD patients induced by difference viral types has not yet been clarified. As one of the few studies with a long-term follow-up design to quantitative determinate the viral load in deference samples of HFMD patients, the results of our research will fill up the gaps in research on the dynamic changes of viral load in patients with HFMD.

The dynamic change process of viral load in the body is affected by many factors including serotypes, specimen types, severity and treatment, thereby affecting the duration of viral shedding [22, 23]. In this study, we will obtain comprehensive data including detailed clinical characteristics, laboratory test results and treatments through prospectively collect and update. And we will quantify the viral load in throat swabs and feces from each patient during the entire detox period through follow-up. The results of our research can further clarify the evolvement rules which reflect the dynamic changes of viral load and the duration of viral shedding in HFMD patients under the influence of related factors.

Moreover, the site of the project is the designated hospital for inpatients with HFMD in Chengdu, and almost all inpatients will be referred to this hospital for treatment. This study will be based on a well-characterized sample of subjects with HFMD, who are assessed for a broad number of predictor variables at baseline including clinical features, laboratory test and treatment. And details of symptoms and signs which may influence outcome will be assessed over the illness course.

There are also undeniable limitations to this study. The major limitation might be the loss of follow-up, just like any other follow up study cannot avoid. Especially because the Clinical Public Health Center of Chengdu is a designated treatment and isolation hospital for COVID-19 patients in Sichuan Province, many patients may be unwilling to return to the hospital for return visits. From this perspective, we will conduct home followup visits by trained project members to complete sample collection. In addition, during the study recruitment process, the investigator will explain that enrollment in the cohort requires continued follow-up. Subjects with a strong willingness to participate will be included, which may avoid loss to follow-up. Appropriate method will be used to deal with the lost data and assess its impact on the results.

On the other hand, the clinical public health center of Chengdu is a regional referral hospital for HFMD management, so some patients might be already on the 2-3th days after the onset of illness when admitted to the hospital. Therefore, for these patients enrolled in the study, the clinical specimens on the first day of illness cannot be obtained. This situation does not influence the observation of virus excretion duration, but it will have a certain impact on the early dynamic changes of viral load. In order to minimize the impact, we will give priority to patients admitted to the hospital on the day of onset during recruitments.

\section{Abbreviations}

HFMD: Hand, foot and mouth disease; EV-A71: Enterovirus A71; CV-A16: Coxsackievirus A16; CV-A6: Coxsackievirus A6; CV-A10: Coxsackievirus A10; STROBE: Strengthening the Reporting of Observational Studies in Epidemiology; BSL-2: Level 2 Biosafety Laboratory; RT-PCR: Reverse transcription polymerase chain reaction. 


\begin{abstract}
Acknowledgements
Firstly, we would like to acknowledge all participants of the study and their families. Secondly, we would like to acknowledge the staff of Chengdu City's Center for Disease Control and Prevention and pediatric Intensive Care Unit (PICU) and pediatric Ward of Infectious Diseases of Chengdu Public Clinical Center for collecting the clinical data and samples of HFMD cases used in this study. Finally, we would like to acknowledge the teachers of the Experimental Center of West China School of Public Health for providing us with experimental equipment and technical consulting services.
\end{abstract}

\section{Authors' contributions}

All authors contributed to the conception and design of the research. Conceptualization, LL and CZH; methodology, DXX, ZCY and ZDL; software, RXL and TXQ; validation, WX and PHX; writing - original draft preparation, DXX and $\mathrm{CZH}$; writing - review and editing, LL, YP and LJ; visualization, HWW; project administration, LL, DXX and ZYL; funding acquisition, LL. All authors have read and agreed to the published version of the manuscript.

\section{Funding}

This research was peer-reviewed and supported by Grants from National Natural Science Foundation of China Young Scientists Fund (Grant No. 81903375). This research was also supported by General Program of China Postdoctoral Science Foundation (Grant No. 2018M643509) and Technology Innovation R\&D Project of Chengdu Science and Technology Bureau (Grant No. 2019-YF05-01080-SN). All funding sources had no role in the design of this study and will not have any role during its execution, analyses, interpretation of the data, or decision to submit results.

\section{Availability of data and materials}

The anonymized datasets generated during the current study will be made available from the corresponding author on reasonable request.

\section{Declarations}

\section{Ethics approval and consent to participate}

The study was approved by the Ethics Review Board of West China School of Public Health (West China Fourth Hospital), Sichuan University. Investigators will strictly abide by the stated commandments of Helsinki and the ethical issues of human biomedical research when conducting this study. Written informed consent will be obtained from all patients' parent or legal guardian and/or themselves. Patient information will remain confidential throughout the study process.

\section{Consent for publication}

Not applicable.

\section{Competing interests}

The authors declare that they have no competing interests.

\section{Author details}

'Department of Epidemiology and Health Statistics, West China School of Public Health and West China Fourth Hospital, Sichuan University, No.16, Section 3, Renmin south road, Wuhou District, Chengdu, Sichuan, China. ${ }^{2}$ Public Health Clinical Center of Chengdu, Chengdu, Sichuan, China. ${ }^{3}$ Department of Microbiology Laboratory, Chengdu Municipal Center for Disease Control and Prevention, Chengdu, Sichuan, China. ${ }^{4}$ Department of Gastroenterology, West China School of Public Health and West China Forth Hospital, Sichuan University, Chengdu, Sichuan, China. ${ }^{5}$ Non-Communicable Diseases Research Center, West China-PUMC C.C. Chen Institute of Health, Sichuan University, Chengdu, Sichuan, China.

Received: 29 January 2022 Accepted: 8 February 2022 Published online: 20 February 2022

\section{References}

1. He SJ, Han JF, Ding XX, Wang YD, Qin CF. Characterization of enterovirus 71 and coxsackievirus A16 isolated in hand, foot, and mouth disease patients in Guangdong, 2010. Int J Infect Dis. 2013;17(11):e1025-1030.
2. Ai Y, Zhang W, Wu J, Zhang J, Shen M, Yao S, Deng C, Li X, Wu D, Tian P, et al. Molecular epidemiology and clinical features of enteroviruses-associated hand, foot, and mouth disease and herpangina outbreak in Zunyi, China, 2019. Front Med. 2021;8:656699.

3. Xie J, Yang X-H, Hu S-Q, Zhan W-L, Zhang C-B, Liu H, Zhao H-Y, Chai H-Y, Chen K-Y, Du Q-Y, et al. Co-circulation of coxsackieviruses A-6, A-10, and A-16 causes hand, foot, and mouth disease in Guangzhou city, China. BMC Infect Dis. 2020;20(1):271

4. Gopalkrishna V, Ganorkar N. Epidemiological and molecular characteristics of circulating CVA16, CVA6 strains and genotype distribution in hand, foot and mouth disease cases in 2017 to 2018 from Western India. J Med Virol. 2021;93(6):3572-80.

5. Lott JP, Liu K, Landry ML, Nix WA, Oberste MS, Bolognia J, King B. Atypical hand-foot-and-mouth disease associated with coxsackievirus A6 infection. J Am Acad Dermatol. 2013;69(5):736-41.

6. Lizasoain A, Mir D, Martínez N, Colina R. Coxsackievirus A10 causing handfoot-and-mouth disease in Uruguay. Int J Infect Dis. 2020;94:1-3.

7. Blomqvist S, Klemola P, Kaijalainen S, Paananen A, Simonen M-L, Vuorinen T, Roivainen M. Co-circulation of coxsackieviruses A6 and A10 in hand, foot and mouth disease outbreak in Finland. J Clin Virol. 2010;48(1):49-54

8. China NHCotPsRo: Overview of national epidemic situation of notifiable infectious diseases in 2019. 2020.

9. Ooi MH, Wong SC, Lewthwaite P, Cardosa MJ, Solomon T. Clinical features, diagnosis, and management of enterovirus 71. Lancet Neurol. 2010;9(11):1097-105.

10. Prevention CCfDCa: Hand, Foot and Mouth Disease Prevention and Control Guidelines (2009 Edition). http://www.chinacdccn/jkzt/crb/bl/szkb/ jszl_2275/200906/t20090612_24707html (2009).

11. NHS: Hand, foot and mouth disease. https://www.nhsuk/conditions/ hand-foot-mouth-disease/ (2018).

12. Li J, Lin C, Qu M, Li X, Gao Z, Zhang X, Liu Y, Huang Y, Wang X, Jia L, et al. Excretion of enterovirus 71 in persons infected with hand, foot and mouth disease. Virol J. 2013;10:31

13. Shu T, Shi-yong Z, Yi W, Qi-min S, Mao-ying J, Da-wei C, Guo-liang X. Observation on virus shedding periods of enterovirus-71 and coxsackievirus A 16 monitored by nucleic acids determination in stool samples of children with hand, foot and mouth disease. Zhonghua er ke za zhi = Chinese journal of pediatrics. 2013;51(10):787-92.

14. Duan X, Chen Z, Li X, Yuan P, Long L. Virus shedding in patients with hand, foot and mouth disease induced by EV71, CA16 or CA6: systematic review and meta-analysis. Pediatr Infect Dis J. 2021;40(4):289-94.

15. Han J, Ma XJ, Wan JF, Liu YH, Han YL, Chen C, Tian C, Gao C, Wang M, Dong XP. Long persistence of EV71 specific nucleotides in respiratory and feces samples of the patients with hand-foot-mouth disease after recovery. BMC Infect Dis. 2010;10:178.

16. Solomon T, Lewthwaite P, Perera D, Cardosa MJ, McMinn P, Ooi MH. Virology, epidemiology, pathogenesis, and control of enterovirus 71. Lancet Infect Dis. 2010;10(11):778-90.

17. von Elm E, Altman DG, Egger M, Pocock SJ, Gøtzsche PC, Vandenbroucke JP. The Strengthening the Reporting of Observational Studies in Epidemiology (STROBE) statement: guidelines for reporting observational studies. Lancet (London, England). 2007;370(9596):1453-7.

18. Qiu Q, Zhou J, Cheng Y, Zhou Y, Liang L, Cui P, Xue Y, Wang L, Wang K, Wang $\mathrm{H}$, et al. Kinetics of the neutralising antibody response in patients with hand, foot, and mouth disease caused by EV-A71: a longitudinal cohort study in Zhengzhou during 2017-2019. EBioMedicine. 2021;68:103398.

19. Joop J. Multilevel analysis: techniques and applications. London: Routledge; 2018.

20. Sissoko D, Duraffour S, Kerber R, Kolie JS, Beavogui AH, Camara AM, Colin G, Rieger T, Oestereich L, Pályi B, et al. Persistence and clearance of Ebola virus RNA from seminal fluid of Ebola virus disease survivors: a longitudinal analysis and modelling study. Lancet Glob Health. 2017;5(1):e80-8.

21. Benchimol El, Smeeth L, Guttmann A, Harron K, Moher D, Petersen I, Sørensen HT, von Elm E, Langan SM. The REporting of studies Conducted using Observational Routinely-collected health Data (RECORD) statement. PLoS Med. 2015;12(10):e1001885.

22. Yanhong W. Preparation, screening and evaluation of pharmacodynamic of antiviral compounds and study on antiviral effects against EV71. Doctor: Whan University; 2013. 
23. Teng $S$, Wei Y, Zhao SY, Lin XY, Shao QM, Wang J. Intestinal detoxification time of hand-foot-and-mouth disease in children with EV71 infection and the related factors. World J Pediatr. 2015;11(4):380-5.

\section{Publisher's Note}

Springer Nature remains neutral with regard to jurisdictional claims in published maps and institutional affiliations.

- fast, convenient online submission

- thorough peer review by experienced researchers in your field

- rapid publication on acceptance

- support for research data, including large and complex data types

- gold Open Access which fosters wider collaboration and increased citations

- maximum visibility for your research: over 100M website views per year

At BMC, research is always in progress.

Learn more biomedcentral.com/submissions 